\title{
Robust Blink Detection Method For Low Frame Rates
}

\author{
Takeshi Toda ${ }^{* a)}$ \\ Member, \\ Kouhei Tsuruoka* \\ Tatsuhiko Miyakawa* Student Member, \\ Xinxin $\mathrm{Liu}^{* *}$ \\ Student Member \\ Non-member
}

(Manuscript received Oct. 9, 2013, revised April 14, 2014)

\begin{abstract}
In recent times, personal mobile devices have become an integral part of daily life. However, since these devices typically demand prolonged durations of focus, they adversely affect a user's blinking rate. Blinking is an essential function of the eye that spreads tears, and thereby, helps avoid eye dryness and soreness. Several blink detection methods have been proposed, however, these methods are unable to effectively monitor involuntary blinks when the camera frame rate is low. To address this issue, this paper proposes a blink detection method for a real-time vision system implemented on the devices that dynamically change the frame rate of the built-in camera according to the usage environment. In the proposed method, the location of eyes is determined and an open-eye template is created by image differencing the initial blinks of the user. Subsequently, the eye is tracked by template matching using the open-eye template in each frame. During eye tracking, the correlation score is analyzed and thresholded with a criterion in order to detect closed eyelids at each frame. Several-frames-of-moving-averaged value of highest correlation score at each frame is used as the threshold value with an offset. In the experimental evaluation, the proposed blink detection method was implemented on a laptop-PC. A total of more than 10,000 true blinks from twenty-three test subjects yielded detection accuracies of 92.2 and $90.1 \%$ at 30 and 6 fps frame rate, respectively.
\end{abstract}

Keywords: visual display terminal (VDT), eye dryness, blink detection, template matching, built-in camera, frame rate

\section{Introduction}

Mobile personal devises (e.g. laptop PCs, smartphones and tablets) are increasingly a part of life and visual display terminal syndrome (so-called VDT syndrome) users rapidly increase at both work and private places in recent years. The most serious symptom of the VDT syndrome is eye dryness. The eye dryness causes the eye strain that in turn encourages the VDT syndrome such as headache, eyestrain, stiff neck and shoulder ${ }^{(1)(2)}$. Because eye blink coats the eye with tears, the eye dryness are worsened by prolonged usages of the eye during the devises operations in which blinking rate is extremely reduced ${ }^{(3)}$. Thus glasses type of blink-promotion system "Wink Glasses" has recently appeared to prevent the eye dryness ${ }^{(4)}$. The system detects user's blink with a sensor and urges the user to blink by clouding up the sheet attached on the lens when there is no blink for five seconds. However the user cannot combine the system with other VDT glasses such as blue light cut glasses that have recently become widespread $^{(5)}$, since the system needs dedicated glasses.

We implemented a blink detection-and-promotion system by real-time image processing on laptop-PC with built-in camera ${ }^{(6)}$. However frame rate of the built-in camera dynamically changed and dropped, and blink detection failure increased. This was because that the blink detection method

a) Correspondence to: Takeshi Toda. E-mail: takeshi.toda@ele.cst. nihon-u.ac.jp

* Nihon University

1-8-14, Surugadai Kanda, Chiyoda-ku, Tokyo 101-8308, Japan

** National Institute of Occupational Saftey and Health, Japan

6-21-1, Nagao, Tama-ku, Kawasaki, Kanagawa 214-8585, Japan we utilized for the system was one of most popular methods which had been studied for communication of patients in serious body paralytics, such as amyotrophic lateral sclerosis (ALS) and multiple sclerosis (MS) ${ }^{(7)-(9)}$. The conventional methods had no consideration of frame rate drop of cameras, because they used high-end cameras and the frame rate was almost $30 \mathrm{fps}$ constant even in dark environment ${ }^{(9)}$. Furthermore, eye-closing duration in the "voluntary" blink is much longer than that of "in-"voluntary blink. We thus needed to investigate new way to overcome the challenge that requires us to detect short duration of blink by low frame rate of camera.

The main contribution of this paper is to provide an involuntary-blink detection method for real-time vision system on the mobile personal devises in which frame rate of camera dynamically changes and drops. The remaining part of the paper is organized as follows. In Sect. 2, the conventional method and the problems is reviewed. In Sect. 3, the details of the proposed method are presented. In Sects. 4 and 5 , experimental method and performance evaluation of the proposed method are described as compared with that of the conventional. Section 5 gives some discussion and Sect. 6 concludes the paper.

\section{Conventional Methods and Problems}

2.1 Conventional Methods Image processing method to judge opening-and-closing state of eyes has been originally studied for preventing dozing off while driving of drivers $^{(10)-(13)}$. Moreover, in recent years, it has been investigated as an interface of computer operation and communication for patients of serious body paralytics, such as ALS 
and MS ${ }^{(7)-(9)(14)}$. There are various image processing methods for the blink detection using template matching ${ }^{(7)-(9)}$ and variance map ${ }^{(14)}$. Most popular method, described here as conventional, is template matching. The conventional studies using the template matching have same processing scheme, where the correlation is computed between an open-eye template and an eye-area-search region in each frame in real time. The correlation value becomes high as the user's eye open and low as the user's eye close. During template matching process, the place where a correlation value is the highest, is judged to be eyes position. And if the correlation value of the place is higher than a threshold value, eyes will be judged to be open. And also if the correlation value of the place is lower than the threshold value, eyes will be judged to be close. The threshold value is optimized through an experiment in advance, and is given with a fixed value.

2.2 Frame Rate Drop In the previous works where high-end cameras were used, the frame rate was almost 30 fps constant even in dark environment ${ }^{(9)}$. On the other hand in the mobile personal devices, the frame rate of the built-in camera drops according to the brightness of usage environment so that the image quality may be maintained. Moreover, the frame rate also falls with the background jobs such as antivirus software. Figure 1 shows measured result of the frame rate variation of a built-in camera on a laptop-PC when room brightness was changed by pulling up-and-down window shades and by turning on-and-off the room lights. SONY VAIO S series 13 was used as the laptop-PC in which CPU was Intel Core i7-3520 (2.9 GHz) with $8 \mathrm{~GB}$ RAM. "EXMOR for PC" CMOS sensor was mounted on the built-in camera, the effective pixels was 1.31 million pixels and maximum frame rate was $30 \mathrm{fps}$. The frame rate was calculated from number of frames captured per second. The frame rate was almost a maximum of $30 \mathrm{fps}$ when the room was bright, and the frame rate fell to $6 \mathrm{fps}$ as the room became dark.

\subsection{Short Duration of Involuntary Blink}

Figure 2(a), (b), (c) and (d) show consecutively captured seven frame images around an involuntary blink when frame rates were $30,19,12$ and $6 \mathrm{fps}$, respectively with the builtin camera. Those specific frame rates were set by changing room brightness as described in previous section. The frame durations were $1 / 30,1 / 20,1 / 13$ and $1 / 7$ seconds, respectively. It turned out that there were three, two and one frames of closing eye images in 30, 19 and $12 \mathrm{fps}$, respectively, but no frames in $6 \mathrm{fps}$.

Conventional systems were designed to detect voluntary blink such as nap of drivers, wink of ALS and MS patients. On the other hand, the involuntary blink targeted here, happens unconsciously according to a mental cause and the eyeclosing duration is much shorter than that of voluntary blink. Figure 3 shows correlation score of the involuntary blink, measured with the laptop-PC with the built-in camera. The correlation score was computed in the template matching between captured open-eye template and eye-area-search region images in each frame at $30 \mathrm{fps}$ of frame rate (described in detail in Sect.3.1). It is confirmed that the correlationscore falling duration corresponding to the blink duration is about 5-6 frames [200-167 milliseconds (msec)]. The duration of the involuntary blink is generally $100-200 \mathrm{msec}$ and is short compared to that of the voluntary blink ${ }^{(15)}$.

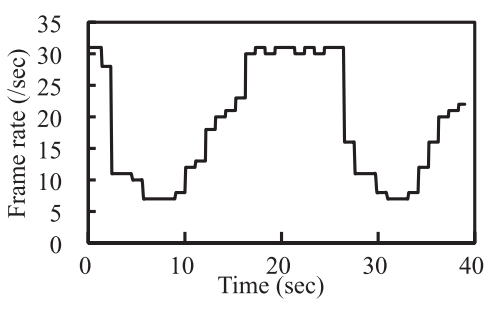

Fig. 1. Frame rate variation of built-in camera on laptop-PC

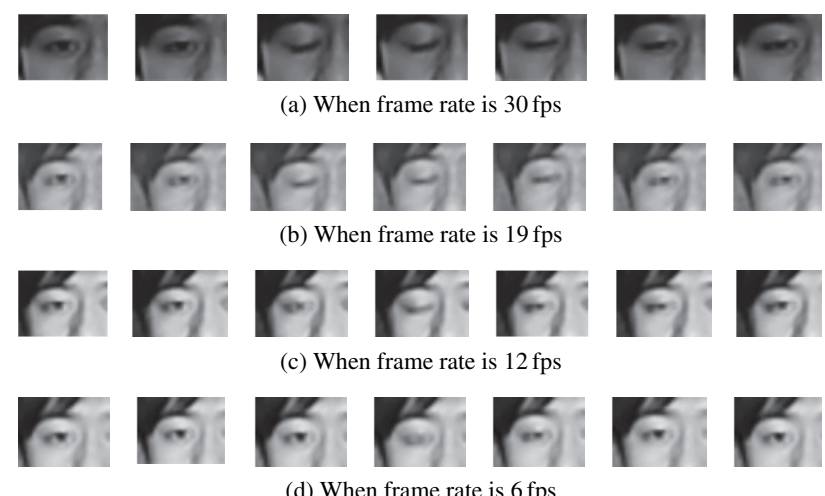

Fig. 2. Captured images of involuntary blinks

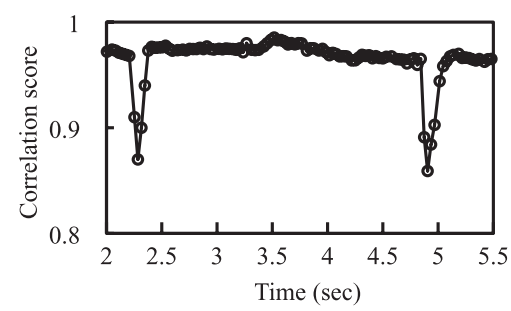

Fig. 3. Template-matching correlation score of involuntary blink

The zero-mean normalized cross-correlation (ZNCC) coefficient which is used in the conventional studies ${ }^{(11)-(13)}$, is utilized for comparison, using following formula:

$$
\begin{aligned}
& \rho(x, y)=\frac{\sum_{u, v}\left[f_{x, y}(x+u, y+v)-\bar{f}_{x, y}\right][t(u, v)-\bar{t}]}{\sqrt{\sum_{u, v}\left[f_{x, y}(x+u, y+v)-\bar{f}_{u, v}\right]^{2}} \sqrt{\sum_{u, v}[t(u, v)-\bar{t}]^{2}}} \\
& \text {................. } \\
& R=\max _{x, y}\{\rho(x, y)\}
\end{aligned}
$$

where $\rho(x, y)$ is the ZNCC coefficient between an open-eye template and an eye-search region images, $R$ is the maximum value of the correlation computed in template matching in each frame. Also $f_{x, y}(x+u, y+v)$ is the brightness of the frame at the reference point $(x+u, y+v)$ in the eye-search region image, $t(u, v)$ is the brightness at the reference point $(u, v)$ in the template image, $\bar{f}_{x, y}$ and $\bar{t}$ are average values of brightness in the eye-search region and the template images respectively. Since both template and search-region images are normalized by average values of brightness respectively, intensity scale is adjusted and the ZNCC was made independent to differences in brightness and contrast, and result in robust against image quality variation.

2.4 Problem in Conventional Method Figure 4(a) and (b) shows correlation characteristics at the frame rate of 


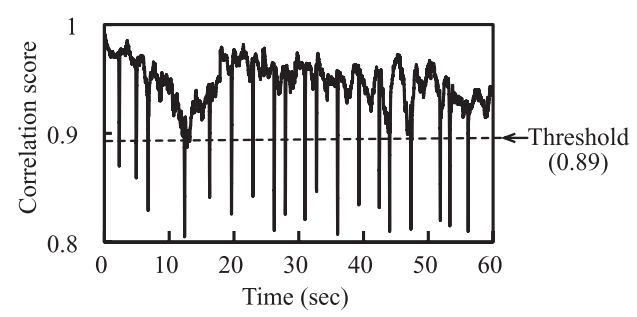

(a) When frame rate is $30 \mathrm{fps}$

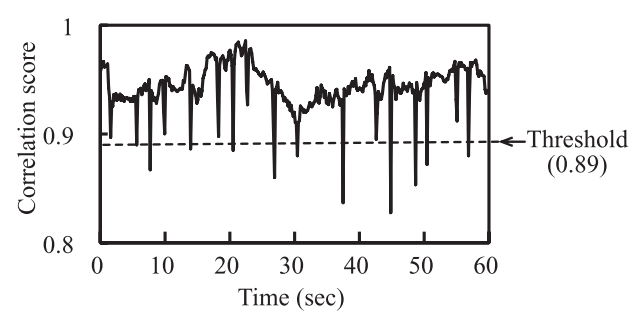

(b) When frame rate is $6 \mathrm{fps}$

Fig. 4. Correlation characteristics changed according to frame rate

30 and 6 fps respectively that were measured and computed using the laptop-PC with the built-in camera same as measurement of correlation score in previous section. The frame rate set $6 \mathrm{fps}$ by darkening room brightness. In the conventional method, blink is judged to occur when the correlation score falls under the threshold value that is optimized through an experiment and is given with a fixed value (0.89). From Fig. 4, it is found out that the variation range of the correlation score becomes narrow and the number of times of the correlation-score drops decreases as the frame rate decreases from 30 to $6 \mathrm{fps}$. Thus missed blinks are supposed to increase as the frame rate decreases. The threshold value needs to be dynamically changed.

\section{Proposed Method}

3.1 Eye Recognition and Tracking Figure 5 shows the flow chart depicting the main stages of the proposed involuntary-blink detection system. The system consists of initial and eye-tracking phases. In the initial phase, the large area of image containing VDT user's face is obtained in each frame. Image difference of each frame and the previous frame is created and then thresholded, resulting in a binary image showing the regions of movement that occurred between the two frames. And the noise of the binary image is rejected by an opening morphological operation and labeling procedure is applied. There are almost no movements in background and body regions other than eye in captured image frame by frame, compared with blink movement in which its change occurs at a speed of several milliseconds. Therefore, when blink occurs, different parts between the two frames are eyes regions and the number of connected components with labeling procedure becomes two (the left and right eyes). However in real, the number of the labeled components may become sometimes two at regions other than eyes, according to influence of surrounding environment and noise, etc. Therefore, the fault resultant can be excluded by applying the experimentally-derived heuristics conditions such as differences of the width, height and distances of both eyes ${ }^{(16)}$. After the just two components are judged to be a pair of the

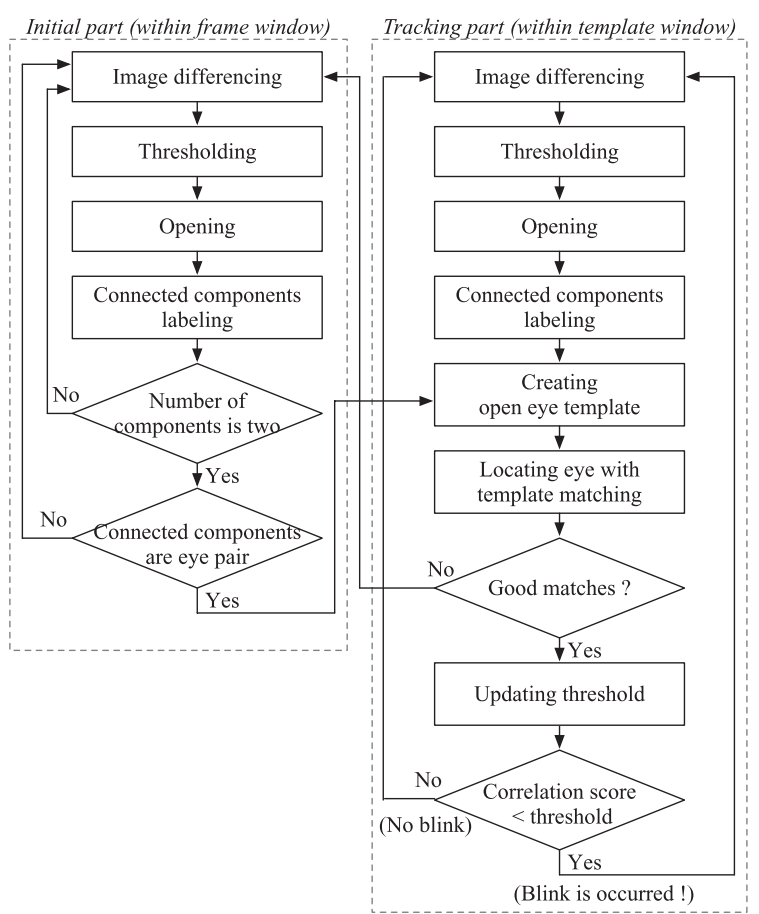

Fig. 5. Proposed in-voluntary blink detection system

right and left eyes, the system procedure moves to next eyetracking phase.

In the eye-tracking phase, the window centering around one-eye (one of two labeled components) is set up to create the open-eye template and also the larger window centering around the pervious window is set up to create eyelocation-search image for conducting template matching in each frame in real time. After the template matching, if the minimum value of the correlation values is larger than a threshold, eye-tracking-phase procedure continues. But if the minimum value of the correlation values is smaller than the threshold, the system procedure returns to the initial phase, being judged to be out of the eye tracking. The eye-tracking method is the same as those of the conventional studies.

3.2 Blink Detection Procedure During eye tracking, blink is judged to occur if the highest value of correlation values is lower than the threshold value at each frame. In the conventional studies, the threshold value is given with a fixed value. On the other hand, in the proposed method, the highest correlation value obtained at each frame is movingaveraged through several frames and an offset is added to the moving-averaged correlation value for every frame, using the following formula:

$$
O_{\text {threshold }}=\left(\frac{1}{M} \sum_{m=1}^{M} R_{m}\right) * \alpha
$$

where $M$ is the number of frames in the moving average, $R_{m}$ is the highest correlation value of template matching in $m$-th frame, and $\alpha$ is the offset coefficient to provide some offset to the correlation value. Slightly offset of the moving-averaged correlation value needs for preventing blink-misdetection that is caused by the correlation-score fluctuating with the influence of surrounding environment and noise, etc. Figure 6 shows the correlation characteristics and the proposed threshold value updated by Eq. (3), where $M$ is five and $\alpha$ is 0.98 


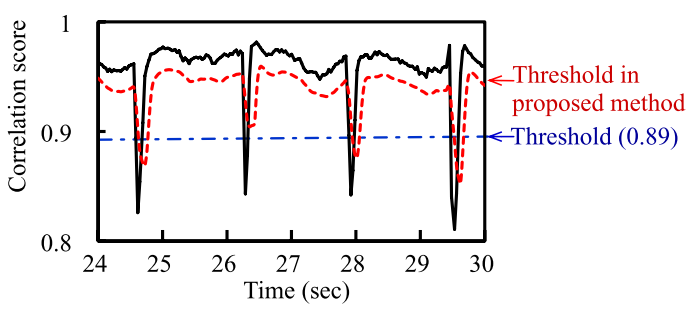

(a) When frame rate is $30 \mathrm{fps}$

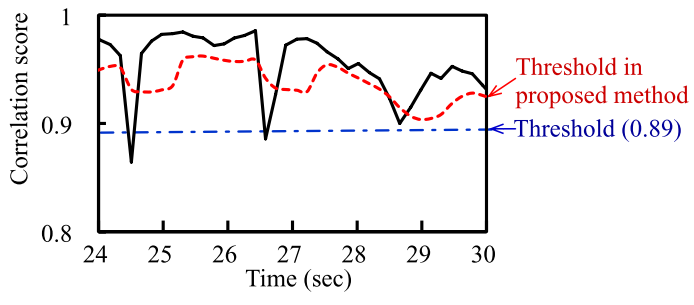

(b) When frame rate is $6 \mathrm{fps}$

Fig. 6. Characteristics of correlation and threshold value changed

when the frame rate is 30 and 6 fps respectively. The fixed threshold (0.89) is also shown by dot-line. It is figured out that the proposed threshold catches three correlation drops (blinks) but the fixed threshold catches two, when the frame rate is $6 \mathrm{fps}$. There are many kinds of thresholding designed in image processing for various purposes such as image binarization and differencing. There are also various methods to dynamically update threshold according to processing image features ${ }^{(17)}$. However, to the best of our knowledge, dynamically thresholding has never been applied to the correlation threshold in the template matching for the blink detection. Furthermore the proposed threshold-updating method using the moving average of the correlation value and the offset is a novel and unique technique for the blink detection procedure.

\section{Experimental Method}

4.1 Specifications of Hardware Both the proposed and conventional blink detection method was implemented on the laptop-PC same as used in previous section (specification is shown in Sect. 2.2). Most VDT used at home and work is laptop-PC and almost equipped with built-in camera. Setup of the display and the camera's setting were left to a setup at the time of factory shipments. Moreover, neither frame rate nor resolution was specially fixed and the specific frame rate was set by changing room brightness as shown in previous sections.

\subsection{Specifications of Blink Detection Method}

Figure 7 shows window sizes of the captured image, eyesearch region, and the eye-template image that are $160 \times 120$, $32 \times 24$, and $16 \times 12$ pixels, respectively. Those window sizes are the same as the conventional studies for comparison. Opening morphological operation is conducted one times using $3 \times 3$ star-shaped convolution kernel. Heuristics conditions for the eye-pair components are also the same as the conventional; difference of component width and height are smaller than five pixels, vertical distance is smaller than five pixels, ratio between horizontal distance and component width is two-to-five pixels, respectively. These specifications are the same as conventional studies.

4.3 Calculating of Blink Detection Accuracy There

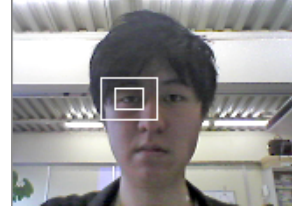

Fig. 7. Eye search and template windows in captured image

are two kinds of blink detection errors that are missed blinks and false-positives. The missed blinks mean blinks that are not detected although the blink occurs. The false-positives means blink is detected although blink dose not occur. Blink detection accuracy $R_{\text {detection }}$ is computed using following formula $^{(12)}$ :

$$
\begin{aligned}
& R_{\text {detection }}=\left(1-\frac{N_{\text {error }}}{N}\right) \ldots \ldots \ldots \ldots \ldots \ldots \ldots \ldots \\
& N_{\text {error }}=N_{\text {missed blinks }}+N_{\text {false-positive }} \ldots \ldots \ldots \ldots \ldots
\end{aligned}
$$

where $N$ is the numbers of true blinks, $N_{\text {error }}$ is the total number of the blink detection error that counts numbers of missed blinks $N_{\text {missed blinks }}$ in addition to false-positives $N_{\text {false-positives }}$. These errors were counted comparing the time-series of blink data logged by the system from that of blink data extracted from the video captured with another camera.

Correlation threshold value to judge blink set 0.89 for the fixed. The number of frames in the moving average of correlation value $(M)$ set five and the offset coefficient $(\alpha)$ was 0.98 respectively for the proposed threshold formulation. These thresholding parameters were optimized before measurement of blink detection accuracy (described in the next chapter in detail).

4.4 Test Subjects A large volume of data was collected in order to examine the effectiveness of the proposed method, compared to the 204 blinks by fifteen subjects measured in Grauman et al. studies ${ }^{(11)(12)}$ and the 2,288 blinks by eight subjects measured in Chau et al. study ${ }^{(13)}$. More than 1,000 true blinks were analyzed respectively for the proposed and conventional methods at each frame rate of 30, 25, 19, 12 and $6 \mathrm{fps}$, and then a total of more than 10,000 true blinks by the twenty-three test subjects were analyzed. The test subjects were all male students from twenty-one to twenty-four years old without eyes glasses. The test subjects adjusted VDT usage-environment freely to himself, such as positions of the chair and VDT, etc.

\section{Result}

\subsection{Optimization of System Parameters}

\section{(a) Fixed Threshold for Conventional Method}

Figure 8 shows the measured result of the blink detection error when the threshold value was changed from 0.87 to 0.9 , at $30 \mathrm{fps}$ of frame rate. About one hundred of true blinks by five subjects were analyzed respectively at each point. From Fig. 8, it is found out that the false-positives decreases and the missed blinks increase as the threshold value decreases. By contraries, the false-positives increases and the missed blinks decreases as the threshold value increases. As the result, 0.89 of the fixed threshold provides minimum number of the total errors. 


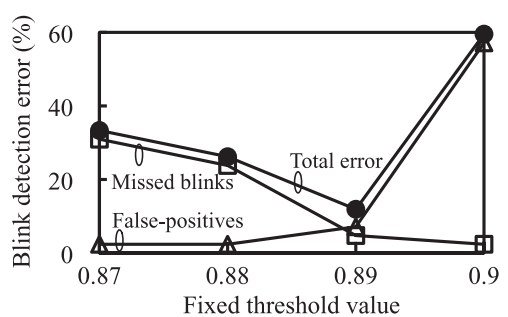

Fig. 8. Blink detection error according to fixed threshold

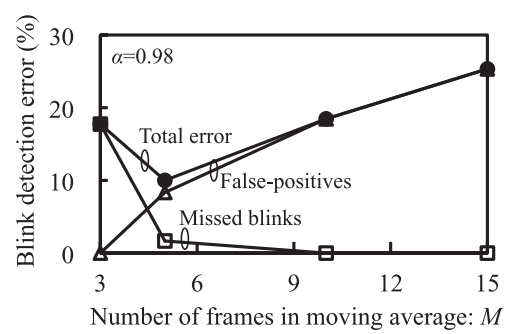

Fig. 9. Blink detection error according to number of frames in moving average of correlation score in proposed method

(b) Number of Frames in Moving Average of Correlation Value for Proposed Method Figure 9 shows the result of blink detection error for proposed method. The number of frames in moving average of the correlation value $(M)$ for the proposed method was changed from 3 to 15 frames. The offset coefficient $(\alpha)$ was 0.98 and the frame rate was 30 fps constant. About one hundred of true blinks by five test subjects were analyzed at each point. From Fig. 9, it is turned out that the false-positives increases and the missed blinks decreased as the number of frames in the moving average increase. This is because that the percentage of the high correlation value in open-eye state increases as the number of frames in the moving average increases and then the moving average of the correlation value becomes higher. As the result, the five-frames of the moving average in the correlation value $(M=5)$ provides minimum number of the total errors.

(c) Offset Coefficient for Proposed Method Figure 10 shows the measured result of the blink detection error for proposed method. The offset coefficient $(\alpha)$ for the proposed thresholding was changed from 0.96 to 0.99 . The number of the frames in moving average was five and the frame rate was $30 \mathrm{fps}$ constant. About one hundred of true blinks by five subjects were analyzed respectively at each point. From the Fig. 10, it is turned out that the missed blinks decreases and the false-positives increases as the offset coefficient closes to one. This is because that the threshold value becomes high as the offset coefficient closes to one. Slightly offset of the moving-averaged correlation value needs for preventing blink-misdetection that is caused by the correlation-score fluctuating with the influence of surrounding environment and noise, etc. From the result, 0.98 of the offset coefficient ( $\alpha=0.98$ ) provides minimum number of the total errors.

5.2 Blink Detection Accuracy Figure 11 shows the measured result of the blink detection accuracy with the proposed method when the frame rate was changed from 30 to 6 , compared to that with the fixed threshold value. The frame rate set by changing the room brightness with pulling up-anddown window shades and turning on-and-off the room lights.

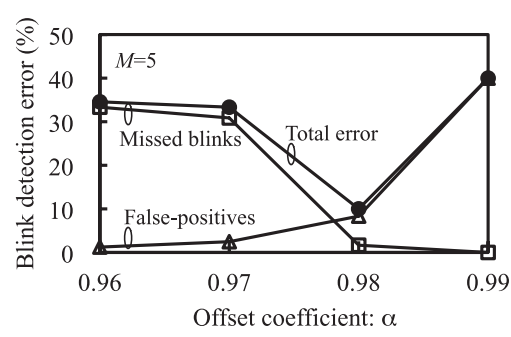

Fig. 10. Blink detection error according to offset coefficient in proposed method

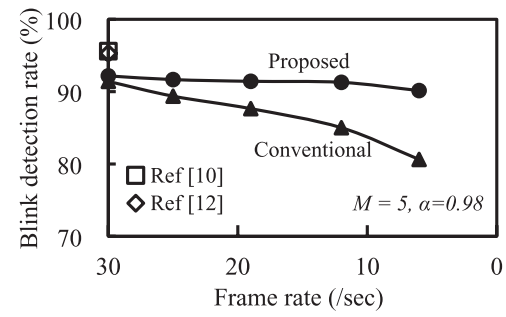

Fig. 11. Blink detection rate according to frame rate

Table 1. Numbers of missed blinks and false-positives

\begin{tabular}{|c|c|c|c|c|c|c|}
\hline \multirow{2}{*}{$\begin{array}{c}\text { Frame } \\
\text { rate }\end{array}$} & \multicolumn{3}{|c|}{\begin{tabular}{c} 
Conventional \\
Total \\
\cline { 3 - 6 }
\end{tabular}} & \multicolumn{2}{|c|}{ Total errors } & \multicolumn{3}{c|}{ Protal } & \multicolumn{2}{c|}{ Total errors } \\
\cline { 7 - 7 } & $\begin{array}{c}\text { Missed } \\
\text { blinks }\end{array}$ & $\begin{array}{c}\text { False } \\
\text {-positives }\end{array}$ & $\begin{array}{c}\text { Total } \\
\text { blinks }\end{array}$ & $\begin{array}{c}\text { Missed } \\
\text { blinks }\end{array}$ & $\begin{array}{c}\text { False } \\
\text {-positives }\end{array}$ \\
\hline \hline 30 & 1037 & 33 & 56 & 1023 & 17 & 63 \\
\hline 25 & 1007 & 51 & 56 & 1008 & 21 & 63 \\
\hline 19 & 1004 & 67 & 57 & 1015 & 22 & 65 \\
\hline 12 & 1001 & 95 & 55 & 1010 & 23 & 65 \\
\hline 6 & 1017 & 139 & 58 & 1015 & 30 & 70 \\
\hline
\end{tabular}

As optimization of the system parameters shown in previous sections, the fixed threshold value set to 0.89 for the conventional, the number of the frames in the moving average of the correlation value set to five and the offset coefficient set to 0.98 for the proposed respectively. Frame rate was kept constant from initial start to finish through each set of the measurement for the blink detection in each frame rate. The measured blink detection accuracies in the previous systems, that are $95.6 \%$ in Grauman et al. studies ${ }^{(10)(11)}$ and the $95.3 \%$ in Chau et al. study ${ }^{(12)}$ at $30 \mathrm{fps}$ of frame rate, are also shown for reference. From the Fig. 11, it is found that the blink detection accuracy of the conventional method worsens from 91.4 to $80.6 \%$ as the frame rate falls from 30 to $6 \mathrm{fps}$. On the other hand, the blink detection accuracy of the proposed method worsens only from 92.2 to $90.1 \%$. Differences of the blink detection accuracy in this system compared to those in the previous systems at the $30 \mathrm{fps}$ of frame rate are thought to be due to experimental setup and conditions such as hardware, environment and the number of tested blinks and test subjects.

The blink detection accuracy is verified by checking the numbers of missed blinks and false-positives respectively in total errors. Table 1 shows the number of missed blinks and false-positives against total number of true blinks respectively in each frame rate. From Table 1, it is confirmed that there are little increase in the false-positives and large increase in the missed blinks for conventional fixed threshold- 
ing, according to the frame rate decreasing. This is because that the variation range of the correlation value becomes narrow and the number of times of deep drop decreases as described in section 2.4. On the other hand, in the proposed thresholding, there was a little increase in both the missed blinks and false-positives as the frame rate decreased. The proposed method quite improved the missed blinks, however could not improve the false-positives for all the frame rates.

\section{Discussion}

The proposed method quite improved the blink detection accuracy that is the total of missed blinks and false-positives, at low frame rates. However the system worsened falsepositives in any of the frame rates, as shown in Table 1 . The system parameters for the thresholding rule, that are fixed threshold value for the conventional method, the number of the frames in the moving average of the correlation value and the offset coefficient for the proposed method, were optimized by about one thousand of true blinks with five test subjects. Thus for either case of the proposed and conventional methods, the missed blinks and false-positives are possibly improved by optimizing those system parameters by much true blinks by much test subjects.

Duration of in-voluntary blinks measured by correlationscore falling was about $167-200 \mathrm{msec}$ as described in Sect. 2.3 and was within value of generally known blink duration $(100-200 \mathrm{msec})^{(15)}$. Assuming a $6 \mathrm{~Hz}$ of periodical signal consecutively occurs in which the $167 \mathrm{msec}(=1 / 6[\mathrm{~Hz}]) \mathrm{du}-$ ration of correlation-score falling caused by blinks, $12 \mathrm{~Hz}$ of sampling rate $(=2 * 1 / 167 \mathrm{msec})$ is required to perfectly record the signal according to the sampling theorem. However here the system was required to just catch the correlation-score point that was lower than a threshold value, in order to detect randomly occurred blinks. This result in the probabilistic problem that obtains four samples from five samples and probability is $80 \%$. By simple thought of stochastic expect, the blink detection accuracy is not beyond of the mark for either case of the conventional and proposed methods.

VDT syndrome is recently becoming more serious for users of the mobile personal devices. A challenge to implement a blink detection system on a smartphone is reported where the frame rate of the built-in camera severely drops by load on the CPU ${ }^{(18)}$. In the study of Han et al. (17), use of the same template matching method as presented here is referred for blink detection, but none of the implementation result is shown. In near future, our proposal described in this paper could be applied to the implementation of the blink detection system on not only the mobile personal devices but also IP cameras in which frame rate falls by network load, etc.

\section{Conclusion}

This paper described needs of monitoring VDT user's "involuntary" blink with the VDT built-in camera for this research background. This paper then focused on a conventional popular image processing method for "voluntary" blink detection by template matching, and implemented the conventional method on a laptop-PC. However, it was found that the frame rate of the VDT built-in camera severely fell according to the environmental brightness and the correlation characteristic in the template matching then changed. With a low frame rate, a fixed value of the thresholding in the template matching for the conventional method caused much missed blinks of the involuntary blink that was quick compared to the voluntary blink.

In order to detect the involuntary blink at a low frame rate, this paper proposed a new blink-detection method by introducing a threshold updating by product of a moving average of the correlation value and an offset coefficient in each frame in real time. For an experimental evaluation, the proposed blink-detection system was implemented on a laptop-PC. A total of more than 10,000 true blink by the twenty-three test subjects were analyzed. The experimental result showed that the blink detection accuracy with the conventional method worsened from 91.4 to $80.6 \%$, and that with the proposed method worsened only from 92.2 to $90.1 \%$, as the frame rate falls from 30 to $6 \mathrm{fps}$.

\section{References}

( 1 ) "Guidelines for industrial health controls of VDT operations", Ministry of Health, Labor and Welfare press release (in Japanese) (2002)

(2) "2009 general condition of the survey result about the Heisei 20 technical innovation and labor", Ministry of Health, Labor and Welfare press release (in Japanese) (2009)

( 3 ) K. Tsubota and K. Nakamori: "Dry eyes and video display terminals", The New England J. Med., Vol.328, No.8, p.584 (1993)

( 4 ) "Wink Glasses", Masunaga Optical Mfg Co., Ltd: http://www.masunaga1905.jp (2013)

( 5 ) Intermestic Inc.: "Zoff PC", http://www.zoff.co.jp (2013)

( 6 ) K. Tsuruoka, T. Toda, and H. Tei: "A proposal of Application for VDT Syndrome Prevention by Blink Promotion in Mobile Computer and Device with Built-in Webcam", Proc. Vision Engineering Workshop (ViEW) 2012, pp.52-53 (in Japanese) (2012)

( 7 ) M. Kaneda and M. Tsukino: "Development of drowsiness detection system", Proc. IEEE Veh. Navi. and Info. Sys. Conf., pp.15-20 (1994)

( 8 ) J.L. Crowley and F. Berard: "Multi-modal tracking of faces for video communications", Proc. IEEE Conf. on Computer Vision and Pattern Recognition (CVPR'97), pp.640-645 (1997)

( 9 ) T. Nakano, K. Sugiyama, M. Mizuno, and S. Yamamoto: "Blink measurement by image processing and application to warning of driver's drowsiness in automobiles", Proc. IEEE Intelligent Vehicles Symposium, pp.285-290 (1998)

(10) Y. Tian, T. Kanade, and J. Cohn: "Dual-state parametric eye tracking”, Proc. IEEE Face and \& Gesture Recognition, pp.110-115 (2000)

(11) K. Grauman, M. Betke, J. Gips, and G. Bradski: "Communication via eye blinks - detection and duration analysis in real time", Proc. IEEE Computer Vision and Pattern Recognition Conf. (CVPR 2001), pp.1010-1017 (2001)

(12) K. Grauman, M. Betke, J. Lombardi, J. Gips, and G. Bradski: "Communication via eye blinks and eyebrow raises: Video-based human-computer interfaces", Universal Access In The Inf. Soc., pp.359-373 (2003)

(13) M. Chau and M. Betke: "Real Time Eye Tracking and Blink Detection with USB Cameras", Boston Univ. Comp. Sci. Tech. Rep., no.2005-12 (2005)

(14) T. Morrisf1, P. Blenkhorn, and F. Zaidi: "Blink detection for real-time eye tracking", J. Net. and Comp. Apps., Vol.25, No.2, pp.129-143 (2002)

(15) H. Tada, T. Yamada, and K. Fukuda: "Psychology of blink", Kitaohji Shobou (in Japanese) (1991)

(16) L.C. Trutoiu, E.J. Carter, I. Matthews, and J.K. Hodgins: "Modeling and animating eye blinks", J. ACM Trans. App. Percept., Vol.8, No.3 (2011)

(17) C. Su and A. Amer: "A Real-Time Adaptive Thresholding for Video Change Detection”, Proc. IEEE Int'l Conf. on Image Processing (2006)

(18) S. Han, S. Yang, J. Kim, and M. Gerla: "EyeGuardian: A Framework of Eye Tracking and Blink Detection for Mobile Device Users", Proc. the 2nd ACM SIGCOMM Workshop on Networking, Systems, and Applications on Mobile Handhelds, pp.15-20 (2012) 
Takeshi Toda (Member) received a B.E. degree in electrical engineer-

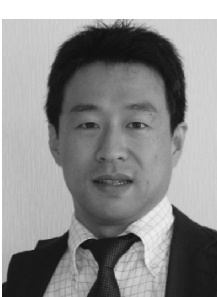
ing from Nihon University, Tokyo, Japan in 1992; an M.S. degree in electronic engineering from the University of Electro-Communications, Tokyo, Japan in 1994; and a D.E. degree from the Tokyo Institute of Technology, Tokyo, Japan in 2004. From 1994 to 2004, he worked at Fujitsu Laboratories Ltd., Kawasaki, Japan. From 2004 to 2005, he worked at eAccess Ltd., Tokyo, Japan. From 2005 to 2008, he worked at Kyocera Corp., R\&D center, Yokohama, Japan. Since 2008, he has been an Assistant Professor at Nihon University, Tokyo, Japan. His current research interests include healthcare and medical support system, etc. He is a member of Japan Ergonomics Society.

Kouhei Tsuruoka (Student Member) received a B.E. and an M.S. de-

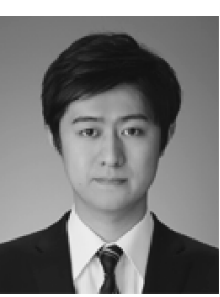
rees in electrical engineering from Nihon University, Tokyo in 2012 and 2014 respectively. Since 2014, he engages in data center operations at SoftBank Corp. $\mathrm{He}$ is a member of Japan Ergonomics Society.
Tatsuhiko Miyakawa (Student Member) received a B.E. degree in

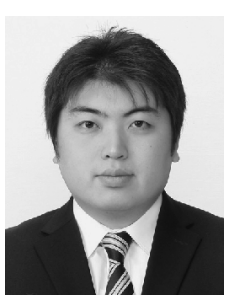
electrical engineering from Nihon University, Tokyo in 2013. He has been working toward an M.S. degree at Nihon University, Tokyo since 2013. His current interests include lifelogging of blink and it's psychological analysis. He is a member of Japan Ergonomics Society.

Xinxin Liu (Non-member) received a B.E. degree in electromechan-

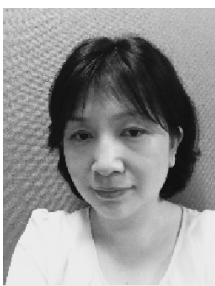
ical engineering from Northeast Forestry University, Harbin, China in 1997; a M.S. degree in humanomics from Chiba University, Chiba, Japan in 2005; and a Ph.D. degree in design science from Chiba University, Chiba, Japan in 2008. From 2008 to 2009, she worked at Chiba University, Chiba, Japan. Since 2010, she has been a researcher at National Institute of Occupational Safety and Health, Japan. Her current research interests include healthcare and stress management, etc. She is a member of Japan Ergonomics Society. 